\title{
Group Analysis and Exact Soliton Solutions to a New $(3+1)$-Dimensional Generalized Kadomtsev-Petviashvili Equation in Fluid Mechanics
}

\author{
Qin Zhou ${ }^{a, *}$, Aimin PAN $^{a}$, Seyed Mehdi Mirhosseini-Allzamini ${ }^{b}$, Mohammad $^{2}$ \\ MirzazAdeH $^{c}$, Wenjun LiU ${ }^{d, \dagger}$ And Anjan Biswas ${ }^{e, f}$ \\ ${ }^{a}$ School of Electronics and Information Engineering Wuhan Donghu University, \\ Wuhan 430212, Peoples Republic of China \\ ${ }^{b}$ Department of Mathematics, Payame Noor University (PNU), P.O. Box 19395-3697, Tehran, Iran \\ ${ }^{c}$ Department of Engineering Sciences, Faculty of Technology and Engineering, East of Guilan, University of Guilan, \\ P.C. 44891-63157, Rudsar-Vajargah, Iran \\ ${ }^{d}$ State Key Laboratory of Information Photonics and Optical Communications and School of Science, \\ P. O. Box 122 Beijing University of Posts and Telecommunications, Beijing 100876, China \\ ${ }^{e}$ Department of Physics, Chemistry and Mathematics, Alabama A\&M University, Normal, AL-35762, USA \\ ${ }^{f}$ Department of Mathematics and Statistics, Tshwane University of Technology, Pretoria-0008, South Africa
}

(Received February 19, 2018; in final form July 9, 2018)

This work studies a new (3+1)-dimensional generalized Kadomtsev-Petviashvili equation analytically. This model is a version of the Kadomtsev-Petviashvili equation that addresses shallow water waves in $(2+1)$-dimensions. Based on the Lie group method, the symmetry reductions and traveling wave reduction are obtained. Finally, explicit solitons including the soliton solutions are constructed by a couple of integration methods, which are the power series approach, subsidiary ordinary differential equation scheme, and the sine-Gordon expansion method.

DOI: 10.12693/APhysPolA.134.564

PACS/topics: solitons, group analysis, symmetry analysis, exact solutions

\section{Introduction}

Ocean dynamics especially in the context of shallow water waves near shores and along beaches are all modeled by various nonlinear evolution equations [1-15]. A few of these well known models that describe the shallow water wave dynamics are the Korteweg-de Vries equation, the Kawahara equation, the Boussinesq equation, the Bona-Chen equation, Gardner's equation and several others. All of these models describe 1-dimensional fluid flow where as the Bona-Chen equation describes the two-layered fluid flow. However, a practical approach to describe this dynamics of shallow water waves is from a two-dimensional perspective. This is described by the Kadomtsev-Petviashvili equation that was proposed earlier. In this context, several results have been reported and the model has been exhaustively studied.

This paper will study a version of the KP equation that has been recently proposed by Wazwaz and El-Tantawy and it was described in $(3+1)$-dimensions in order to look at it from a generalized setting [1]. The Lie group analysis as well as Frobenius' series solution method, traveling

\footnotetext{
*corresponding author; e-mail: qinzhou@whu.edu.cn

${ }^{\dagger}$ corresponding author; e-mail: jungliu@bupt.edu.cn
}

wave hypothesis, sub ordinary differential equation (subODE) method and the sine-Gordon expansion method are the integrability approaches that are adopted in this paper. These algorithms reveal solitary waves, shock waves and other solutions to the model that are novel contributions in the field of ocean engineering. This new model reads as

$$
u_{t x}+u_{t y}+u_{t z}-u_{z z}+u_{x x x y}+3\left(u_{x} u_{y}\right)_{x}=0 .
$$

The rest of the paper will focus on the integrability aspects of this paper using the Lie symmetry, traveling wave hypothesis, series solutions approach, sub-ODE scheme and the sine-Gordon expansion method. The details are in subsequent sections.

\section{Lie symmetry analysis}

In this section, we consider Eq. (1) using the Lie group method. On the basis of the Lie group method, one can get

$$
\begin{aligned}
& \xi_{t}=\left(c_{1}-c_{2}\right) t+c_{4}, \\
& \xi_{x}=\frac{c_{1}(8 t+2 x+4 z)}{6}+\frac{c_{2}(-4 t-x+z)}{6}+c_{6}, \\
& \xi_{y}=\frac{c_{2}(-y+z)}{2}+c_{1} y+c_{5}, \\
& \xi_{z}=c_{1} z+c_{2} t+c_{3},
\end{aligned}
$$




$$
\begin{aligned}
& \eta_{u}=F_{1}(t)+F_{2}(t+z)+\frac{c_{1}(-6 u+8 x+8 y)}{18} \\
& +\frac{c_{2}(3 u-4 x-4 y)}{18}
\end{aligned}
$$

where $F_{1}(t)$ is arbitrary function of $t$, and $F_{2}(t+z)$ are arbitrary functions of $t+z$.

One can get the symmetries as follows:

$$
\begin{aligned}
& V_{1}=\partial_{t}, \quad V_{2}=\partial_{x}, \quad V_{3}=\partial_{y}, \quad V_{4}=\partial_{z}, \\
& V_{5}=-t \partial_{t}+\frac{-4 t-x+z}{6} \partial_{x}+\frac{-y+z}{2} \partial_{y} \\
& +t \partial_{z}+\frac{3 u-4 x-4 y}{18} \partial_{u}, \\
& V_{6}=t \partial_{t}+\frac{8 t+2 x+4 z}{6} \partial_{x}+y \partial_{y}+z \partial_{z} \\
& +\frac{-6 u+8 x+8 y}{18} \partial_{u}, \\
& V_{F_{1}}=F_{1} \partial_{u}, \quad V_{F_{2}}=F_{2} \partial_{u} .
\end{aligned}
$$

\section{Symmetry reductions}

In this section, we consider the symmetry reductions.

$$
\text { 3.1. } V_{1}
$$

For this case, we get the invariants are $\xi=x, \eta=y, \theta=z$. Therefore, the invariant function is $f(\xi, \eta, \theta)=u(x, y, z)$. Therefore, one can get the following equation:

$$
-f_{\theta \theta}+f_{\xi \xi \xi \eta}+3 f_{\xi \xi} f_{\eta}+3 f_{\xi} f_{\xi \eta}=0 .
$$

It is clear that this equation still is a nonlinear partial differential equation (PDE). Once again, we consider the symmetry using the Lie group again. One has

$$
\begin{aligned}
& \Gamma_{\xi}=\frac{\left(2 c_{3}-c_{1}\right) \xi}{3}+c_{5}, \\
& \Gamma_{\eta}=c_{1} \eta+c_{2}, \\
& \Gamma_{\theta}=c_{3} \theta+c_{4}, \\
& \Gamma_{f}=\frac{\left(-2 c_{3}+c_{1}\right) f}{3}+c_{6} \theta+c_{7} .
\end{aligned}
$$

For this equation, we have the following symmetries:

$$
\begin{aligned}
& V_{1}=\partial_{\xi}, \quad V_{2}=\partial_{\eta}, \quad V_{3}=\partial_{\theta}, \quad V_{4}=\partial_{f}, \quad V_{5}=\theta \partial_{f}, \\
& V_{6}=\frac{2 \xi}{3} \partial_{\xi}-\frac{2 f}{3} \partial_{f}, \quad V_{7}=-\frac{\xi}{3} \partial_{\xi}=\frac{f}{3} \partial_{f} .
\end{aligned}
$$

\section{2. $V_{2}$}

For this case, we get the following equations:

$$
f_{\tau \eta}+f_{\tau \theta}-f_{\theta \theta}=0 .
$$

where the invariants are $\tau=t, \eta=y, \theta=z$ and the invariant function is $f(\tau, \eta, \theta)=u(t, y, z)$. It is a linear
PDE. Once again, we consider the symmetry using Lie group again. One has

$$
\begin{aligned}
\Gamma_{f} & =\frac{1}{6} u\left(8 t c_{1}-2 y c_{1}+2 z c_{1}-3 y c_{5}+3 z c_{5}\right. \\
& \left.-12 z c_{8}+6 c_{11}\right)+F_{3}, \\
\Gamma_{\eta} & =\frac{2}{3} z^{2} c_{1}+c_{3}+y^{2} c_{5}+y\left(c_{4}+z\left(\frac{2 c_{1}}{3}-c_{5}+4 c_{8}\right)\right) \\
& +z\left(c_{2}-2 c_{7}+c_{10}\right), \\
\Gamma_{\theta} & =\frac{1}{6}\left(-2 y^{2} c_{1}+3 y^{2} c_{5}-12 y^{2} c_{8}\right. \\
& \left.+z\left(3\left(2 c_{2}+c_{4}-z\left(2 c_{1}+c_{5}-4 c_{8}\right)\right)+8 y\left(c_{1}+3 c_{8}\right)\right)\right) \\
& +\frac{1}{6}\left(6 c_{9}+6 y c_{10}+4 t\left(-4 z c_{1}+y\left(2 c_{1}-3 c_{5}+12 c_{8}\right)\right.\right. \\
& \left.\left.+3\left(c_{2}-2 c_{7}+c_{10}\right)\right)\right), \\
\Gamma_{\tau} & =\frac{1}{12}\left(-32 t^{2} c_{1}+3\left(c_{3}-z c_{4}+4 c_{6}+4 z c_{7}+4 y^{2} c_{8}\right.\right. \\
& \left.\left.+4 z^{2} c_{8}+y\left(c_{4}-4 c_{7}-8 z c_{8}\right)-2 c_{9}\right)\right) \\
& -\frac{1}{12}\left(4 t \left(z\left(2 c_{1}+3 c_{5}-12 c_{8}\right)+y\left(-2 c_{1}-3 c_{5}+12 c_{8}\right)\right.\right. \\
& \left.\left.+3\left(c_{2}-6 c_{7}+3 c_{10}\right)\right)\right) .
\end{aligned}
$$

In addition we have the constraint conditions

$$
F_{3_{\theta \tau}}-F_{3_{\theta \theta}}+F_{3_{\eta \tau}}=0 .
$$

\section{3. $V_{4}$}

In this case, the invariants are $\xi=x, \eta=y, \tau=t$ and the invariant function is $f(\xi, \eta, \tau)=u(x, y, t)$. Thus, one can get the following equation:

$$
f_{\xi \tau}+f_{\eta \tau}+f_{\xi \xi \xi \eta}+3 f_{\xi \xi} f_{\eta}+3 f_{\xi} f_{\xi \eta}=0 .
$$

As the same previous step, we obtain

$$
\begin{aligned}
& \Gamma_{\xi}=\frac{c_{1} \xi}{3}+c_{3} t+c_{4}, \\
& \Gamma_{\eta}=\frac{c_{1}}{3} \eta+c_{5}, \\
& \Gamma_{\tau}=c_{1} \tau+c_{2}, \\
& \Gamma_{f}=\frac{(x+y) c_{3}}{3}-\frac{c_{1} f}{3}+F_{4}(t),
\end{aligned}
$$

where $F_{4}(t)$ is arbitrary function of $t$. For this equation, we have the following symmetries:

$$
V_{1}=\partial_{\xi}, V_{2}=\partial_{\eta}, V_{3}=\partial_{\tau}, V_{F}=F_{4} \partial_{f},
$$

$$
\begin{aligned}
V_{4} & =\frac{\xi}{3} \partial_{\xi}+\frac{\eta}{3} \partial_{\eta}+\tau \partial_{\tau}-\frac{f}{3} \partial_{f}, \\
V_{5} & =t \partial_{\xi}+\frac{x+y}{3} \partial_{f} .
\end{aligned}
$$




\section{Traveling wave reduction}

In this section the governing Eq. (1) will be studied with the help of traveling wave hypothesis with general coefficients. This allows addressing (1) from a generalized perspective. Therefore, the generalized form of (1) that will be focused in this section will be

$$
\begin{aligned}
& a_{1} u_{t x}+a_{2} u_{t y}+a_{3} u_{t z}+b_{1} u_{z z}+b_{2} u_{x x x y} \\
& +c\left(u_{x} u_{y}\right)_{x}=0 .
\end{aligned}
$$

To study (23), the following traveling wave hypothesis is selected:

$$
u(x, y, z, t)=g\left(B_{1} x+B_{2} y+B_{3} z-v t\right)=g(s),
$$

where

$$
s=B_{1} x+B_{2} y+B_{3} z-v t .
$$

Substituting (24) into (23) and integrating leads to

$$
\begin{aligned}
& b_{2} B_{1}^{3} B_{2} h^{\prime \prime}=\left(v a_{1} B_{1}+v a_{2} B_{2}+v a_{3} B_{3}-b_{1} B_{3}^{2}\right) h \\
& \quad-c B_{1}^{2} B_{2} h^{2},
\end{aligned}
$$

where the notations $h^{\prime}=\mathrm{d} h / \mathrm{d} s, h^{\prime \prime}=d^{2} h / d s^{2}$ with $g^{\prime}=h$ are adopted. Next, multiplying both sides of () by $h^{\prime}$ and integrating gives

$h^{\prime}=\sqrt{\frac{2 c}{3 b_{2}}\left(\frac{3\left(v a_{1} B_{1}+v a_{2} B_{2}+v a_{3} B_{3}-b_{1} B_{3}^{2}\right)}{2 c B_{1}^{2} B_{2}}-h\right)} h$

Integration of (27) and transforming back to its original variables leads to the shock wave solution

$u(x, y, z, t)=A \tanh \left[B\left(B_{1} x+B_{2} y+B_{3} z-v t\right)\right]$,

where parameters $A$ and $B$ are given by

$A=\frac{3}{c B_{1}} \sqrt{\frac{b_{2}}{B_{2}}\left(v a_{1} B_{1}+v a_{2} B_{2}+v a_{3} B_{3}-b_{1} B_{3}^{2}\right)}$

$B=\frac{1}{2 B_{1}} \sqrt{\frac{v a_{1} B_{1}+v a_{2} B_{2}+v a_{3} B_{3}-b_{1} B_{3}^{2}}{b_{2} B_{2}}}$.

These shock wave solutions will exist provided

$$
c B_{1} \neq 0
$$

and

$$
b_{2} B_{2}\left(v a_{1} B_{1}+v a_{2} B_{2}+v a_{3} B_{3}-b_{1} B_{3}^{2}\right)>0 .
$$

\section{Exact solutions}

5.1. Exact solution with power series method

In this section, we deal with the exact solutions of (24). We can get the solutions of other equations in the same way. Now, we assume Eq. (1) has a solution as follows:

$$
f(\xi)=c_{0}+c_{1} \xi+c_{2} \xi^{2}+\cdots=\sum_{n=0}^{\infty} c_{n} \xi^{n} .
$$

Substituting (33) into (1), one has

$$
\begin{gathered}
(-3 c-1) c_{1}+(-3 c-1) \sum_{n=1}^{\infty}(n+1) c_{n+1} \xi^{n} \\
+3 c_{1}^{2}+3 \sum_{n=1}^{\infty} \sum_{j=0}^{n+1} j(n+2-j) c_{j} c_{n+2-j} \xi^{n}
\end{gathered}
$$

$$
+6 c_{3}+\sum_{n=1}^{\infty}(n+3)(n+2)(n+1) c_{n+3} \xi^{n}=0 .
$$

We compare coefficients for $n=0$ in (34), one obtains

$$
c_{3}=\frac{(3 c+1) c_{1}-3 c_{1}^{2}}{6}
$$

In general, for $n \geq 1$, one has

$c_{n+3}=$

$$
\frac{(-3 c-1)(n+1) c_{n+1}+3 \sum_{j=0}^{n+1} j(n+2-j) c_{j} c_{n+2-j}}{(n+1)(n+2)(n+3)} .
$$

In this case, the power series solution becomes

$$
\begin{aligned}
& f(\xi)=c_{0}+c_{1} \xi+c_{2} \xi^{2} \\
& +c_{3} \xi^{3}+\sum_{n=1}^{\infty} c_{n+3} \xi^{n+3}= \\
& c_{0}+c_{1} \xi+c_{2} \xi^{2}+\frac{(3 c+1) c_{1}-3 c_{1}^{2}}{6} \xi^{3} \\
& +\sum_{n=1}^{\infty} \frac{1}{(n+1)(n+2)(n+3)} \\
& \quad \times\left((-3 c-1)(n+1) c_{n+1}\right. \\
& \left.\quad+3 \sum_{j=0}^{n+1} j(n+2-j) c_{j} c_{n+2-j}\right) \xi^{n+3} .
\end{aligned}
$$

Then, putting $\xi=x+y+z-c t$ into above equation, one can get

$$
\begin{aligned}
& u(x, y, z, t)=c_{0}+c_{1}(x+y+z-c t)+c_{2}(x+y+z-c t)^{2} \\
& +c_{3}(x+y+z-c t)^{3}+\sum_{n=1}^{\infty} c_{n+3}(x+y+z-c t)^{n+3}= \\
& c_{0}+c_{1}(x+y+z-c t)+c_{2}(x+y+z-c t)^{2} \\
& +\frac{(3 c+1) c_{1}-3 c_{1}^{2}}{6}(x+y+z-c t)^{3} \\
& +\sum_{n=1}^{\infty} \frac{1}{(n+1)(n+2)(n+3)}\left((-3 c-1)(n+1) c_{n+1}\right. \\
& \left.+3 \sum_{j=0}^{n+1} j(n+2-j) c_{j} c_{n+2-j}\right)(x+y+z-c t)^{n+3},
\end{aligned}
$$

where $c_{i}(i=0,1,2,3)$ are arbitrary constants.

\subsection{Exact solutions by sub-ODE method}

Based on the sub-ODE method [8], we can assume that Eq. (1) has the following solutions:

$$
f(\xi)=a_{0}+a_{1} \varphi \text {. }
$$

Here, we need to solve $a_{0}, a_{1}$, At the same time, $\varphi(\xi)$ needs to be satisfied by the following equation:

$$
\varphi^{\prime}=A+B \varphi+C \varphi^{2} .
$$

Substituting (33) with (34) into (32), and collecting 
different coefficients for all of $\varphi$, then setting the coefficients equal to zero, we have

$$
\begin{aligned}
& 4 A C-B^{2}=-3 c-1, \quad B=B, \quad C=C, \quad c=c, \\
& a_{1}=-2 C, \quad a_{0}=a_{0} .
\end{aligned}
$$

It is well known that Eq. (38) has many solutions. We can get exact solutions of Eq. (1) via solutions of Eq. (38).

Family 1: When $\Delta=B^{2}-4 A C>0$ and $B C \neq 0$ (or $A C \neq 0)$,

$$
\begin{aligned}
& u(x, t)=a_{0}-a_{1} \frac{1}{2 C}\left[B+\Delta \tanh \left(\frac{\Delta}{2} \xi\right)\right], \\
& u(x, t)=a_{0}-a_{1} \frac{1}{2 C}\left[B+\Delta \operatorname{coth}\left(\frac{\Delta}{2} \xi\right)\right], \\
& u(x, t) \\
& =a_{0}-a_{1} \frac{1}{2 C}[B+\Delta(\tanh (\Delta \xi) \pm \mathrm{i} \operatorname{sech}(\Delta \xi))] \\
& u(x, t)= \\
& a_{0}-a_{1} \frac{1}{2 C}[B+\Delta(\operatorname{coth}(\Delta \xi) \pm \mathrm{i} \operatorname{csch}(\Delta \xi))], \\
& u(x, t)= \\
& a_{0}-a_{1} \frac{1}{4 C}\left[2 B+\Delta\left(\tanh \left(\frac{\Delta}{4} \xi\right)+\operatorname{coth}\left(\frac{\Delta}{4} \xi\right)\right)\right] \\
& u(x, t)= \\
& a_{0}+a_{1} \frac{1}{2 C}\left[-B+\frac{\sqrt{\left(E^{2}+F^{2}\right)} \Delta-E \Delta \cosh (\Delta \xi)}{E \sinh (\Delta \xi)+F}\right], \\
& u(x, t)= \\
& a_{0}+a_{1} \frac{1}{2 C}\left[-B-\frac{\sqrt{\left(F^{2}-E^{2}\right)} \Delta+E \Delta \sinh (\Delta \xi)}{E \cosh (\Delta \xi)+F}\right],
\end{aligned}
$$

where $E$ and $F$ are two non-zero real constants and satisfy $F^{2}-E^{2}>0$.

$$
\begin{aligned}
& u(x, t)=a_{0}+a_{1}\left[\frac{2 A \cosh \left(\frac{\Delta}{2} \xi\right)}{\Delta \sinh \left(\frac{\Delta}{2} \xi\right)-B \cosh \left(\frac{\Delta}{2} \xi\right)}\right], \\
& u(x, t)=a_{0}+a_{1}\left[\frac{-2 A \sinh \left(\frac{\Delta}{2} \xi\right)}{-\Delta \cosh \left(\frac{\Delta}{2} \xi\right)+B \sinh \left(\frac{\Delta}{2} \xi\right)}\right], \\
& u(x, t)=a_{0}+a_{1}\left[\frac{2 A \cosh (\Delta \xi)}{\Delta \sinh (\Delta \xi)-B \cosh (\Delta \xi) \pm \mathrm{i} \Delta}\right], \\
& u(x, t)=a_{0}+a_{1}\left[\frac{2 A \sinh (\Delta \xi)}{\Delta \cosh (\Delta \xi)-B \sinh (\Delta \xi) \pm \Delta}\right], \\
& u(x, t)= \\
& a_{0}+a_{1}\left[\frac{4 A \sinh \left(\frac{\Delta}{4} \xi\right) \cosh \left(\frac{\Delta}{4} \xi\right)}{-2 B \sinh \left(\frac{\Delta}{4} \xi\right) \cosh \left(\frac{\Delta}{4} \xi\right)+2 \Delta \cosh ^{2}\left(\frac{\Delta}{4} \xi\right)-\Delta}\right] .
\end{aligned}
$$

Family 2: When $\Delta=4 A C-B^{2}>0$ and $B C \neq 0$ (or $A C \neq 0)$,

$$
u(x, t)=a_{0}+a_{1} \frac{1}{2 C}\left[-B+\Delta \tan \left(\frac{\Delta}{2} \xi\right)\right],
$$

$$
\begin{gathered}
u(x, t)=a_{0}-a_{1} \frac{1}{2 C}\left[B+\Delta \cot \left(\frac{\Delta}{2} \xi\right)\right], \\
u(x, t)= \\
a_{0}+a_{1} \frac{1}{2 C}[-B+\Delta(\tan (\Delta \xi) \pm \sec (\Delta \xi))], \\
u(x, t)=a_{0}-a_{1} \frac{1}{2 C}[B+\Delta(\cot (\Delta \xi) \pm \csc (\Delta \xi))], \\
u(x, t)= \\
a_{0}-a_{1} \frac{1}{4 C}\left[-2 B+\Delta\left(\tan \left(\frac{\Delta}{4} \xi\right)-\cot \left(\frac{\Delta}{4} \xi\right)\right)\right], \\
u(x, t)= \\
a_{0}+a_{1} \frac{1}{2 C}\left[-B+\frac{ \pm \sqrt{\left(F^{2}-E^{2}\right)} \Delta-E \Delta \cos (\Delta \xi)}{E \sin (\Delta \xi)+F}\right], \\
u(x, t)= \\
a_{0}+a_{1} \frac{1}{2 C}\left[-B+\frac{ \pm \sqrt{\left(F^{2}-E^{2}\right)} \Delta+E \Delta \sinh (\Delta \xi)}{E \cos (\Delta \xi)+F}\right],
\end{gathered}
$$

where $E$ and $F$ are two non-zero real constants and satisfy $F^{2}-E^{2}>0$.

$$
\begin{aligned}
& u(x, t)=a_{0}+a_{1}\left[\frac{-2 A \cos \left(\frac{\Delta}{2} \xi\right)}{\Delta \sin \left(\frac{\Delta}{2} \xi\right)+B \cos \left(\frac{\Delta}{2} \xi\right)}\right], \\
& u(x, t)=a_{0}+a_{1}\left[\frac{2 A \sin \left(\frac{\Delta}{2} \xi\right)}{\Delta \cos \left(\frac{\Delta}{2} \xi\right)-B \sin \left(\frac{\Delta}{2} \xi\right)}\right], \\
& u(x, t)=a_{0}+a_{1}\left[\frac{-2 A \cos (\Delta \xi)}{\Delta \sin (\Delta \xi)+B \cos (\Delta) \pm \Delta}\right], \\
& u(x, t)=a_{0}+a_{1}\left[\frac{2 A \sin (\Delta \xi)}{\Delta \cos (\Delta \xi)-B \sin (\Delta) \pm \Delta}\right], \\
& u(x, t)= \\
& a_{0}+a_{1}\left[\frac{4 A \sin \left(\frac{\Delta}{4} \xi\right) \cos \left(\frac{\Delta}{4} \xi\right)}{-2 B \sin \left(\frac{\Delta}{4} \xi\right) \cos \left(\frac{\Delta}{4} \xi\right)+2 \Delta \cos ^{2}\left(\frac{\Delta}{4} \xi\right)-\Delta}\right] .
\end{aligned}
$$

Family 3: When $A=0$ and $B C \neq 0$,

$$
\begin{aligned}
& u(x, t)=a_{0}+a_{1}\left(\frac{-B d}{C(d+\cosh (B \xi)-\sinh (B \xi))}\right), \\
& u(x, t)=a_{0}+a_{1}\left(-\frac{\cosh (B \xi)+\sinh (B \xi)}{C(d+\cosh (B \xi)+\sinh (B \xi))}\right),
\end{aligned}
$$

where $d$ is an arbitrary constant.

Family 4: When $A=B=0$ and $C \neq 0$,

$$
u(x, t)=a_{0}+a_{1}\left(\frac{-1}{B \xi+k}\right),
$$

where $k$ is an arbitrary constant.

Remark 1: As a matter of fact, these solutions are got from the following equation [7]: $\varphi=$

$$
\frac{\sqrt{4 A C-B^{2}}}{2 C} \frac{C_{1} \mathrm{e}^{\frac{\theta}{2} \sqrt{4 A C-B^{2}}}-C_{2} \mathrm{e}^{-\frac{\theta}{2} \sqrt{4 A C-B^{2}}}}{C_{1} \mathrm{e}^{\frac{\theta}{2} \sqrt{4 A C-B^{2}}}+C_{2} \mathrm{e}^{-\frac{\theta}{2} \sqrt{4 A C-B^{2}}}}-\frac{B}{2 C},
$$

where $C_{1}, C_{2}$ are arbitrary constants. However, from the physical point, these solutions may have different meanings. 


\subsection{Exact solution with sine-Gordon expansion method}

Let us start under the assumption that travelling wave transformation of Eq. (1) is of the form

$$
u(x, y, z, t)=U(\xi), \quad \xi=k_{1} x+k_{2} y+k_{3} z-\nu t,
$$

where $k_{i}(i=1,2,3)$ and $\nu$ are constants, all of them are to be determined. Substituting this hypothesis into Eq. (1), we obtain

$$
\begin{aligned}
& k_{1}^{3} k_{2} U^{(4)}+6 k_{1}^{2} k_{2} U^{\prime} U^{\prime \prime} \\
& \quad-\left(\nu k_{1}+\nu k_{2}+\nu k_{3}+k_{3}^{2}\right) U^{\prime \prime}=0 .
\end{aligned}
$$

Integrating (67) once with respect to $\xi$ and choosing constant of integration to be zero without any loss of generality, we arrive at the ordinary differential equation

$$
\begin{aligned}
& k_{1}^{3} k_{2} U^{\prime \prime \prime}+3 k_{1}^{2} k_{2}\left(U^{\prime}\right)^{2} \\
& \quad-\left(\nu k_{1}+\nu k_{2}+\nu k_{3}+k_{3}^{2}\right) U^{\prime}=0 .
\end{aligned}
$$

In this context, sine-Gordon expansion method will be applied to Eq. (1).

Balancing the terms $U^{\prime \prime \prime}$ and $\left(U^{\prime}\right)^{2}$ by using homogeneous principle yields $N=1$. Thus, the solution has the form

$$
U(\xi)=B_{1} \operatorname{sech}(\xi)+A_{1} \tanh (\xi)+A_{0},
$$

and therefore

$$
U(w)=B_{1} \sin (w)+A_{1} \cos (w)+A_{0},
$$

where either $A_{1}$ or $B_{1}$ may be zero, but both $A_{1}$ or $B_{1}$ cannot be zero simultaneously.

By substituting Eq. (70) into Eq. (68) and simplifying, we obtain the following nonlinear algebraic system:

$$
\begin{aligned}
& k_{1}^{3} k_{2} B_{1} \sin (w) \cos ^{3}(w)+\left(-4 k_{1}^{3} k_{2} A_{1}+\right. \\
& \left.3 k_{1}^{2} k_{2} B_{1}^{2}\right) \sin ^{2}(w) \cos ^{2}(w)+\left(-5 k_{1}^{3} k_{2} B_{1}\right. \\
& \left.-6 k_{1}^{2} k_{2} B_{1} A_{1}\right) \sin ^{3}(w) \cos (w)+\left(2 k_{1}^{3} k_{2} A_{1}\right. \\
& \left.+3 k_{1}^{2} k_{2} A_{1}^{2}\right) \sin ^{4}(w)+\left(-B_{1} \nu\left(k_{1}+k_{2}+k_{3}\right)\right. \\
& \left.-B_{1} k_{3}^{2}\right) \sin (w) \cos (w)+\left(A_{1} \nu\left(k_{1}+k_{2}+k_{3}\right)\right. \\
& \left.+A_{1} k_{3}^{2}\right) \sin ^{2}(w)=0 .
\end{aligned}
$$

Setting each summation of the coefficients of the trigonometric identities of like powers to zero, we recover the following algebraic system of equations:

$\sin (w) \cos (w)$ :

$$
k_{1}^{3} k_{2} B_{1}-B_{1} \nu\left(k_{1}+k_{2}+k_{3}\right)-B_{1} k_{3}^{2}=0,
$$

$\sin ^{3}(w) \cos (w):$

$$
-k_{1}^{3} k_{2} B_{1}-5 k_{1}^{3} k_{2} B_{1}-6 k_{1}^{2} k_{2} B_{1} A_{1}=0,
$$

$\sin ^{2}(w):$

$$
-4 k_{1}^{3} k_{2} A_{1}+3 k_{1}^{2} k_{2} B_{1}^{2}+A_{1} \nu\left(k_{1}+k_{2}+k_{3}\right)+A_{1} k_{3}^{2}=0,
$$

$\sin ^{4}(w):$

$$
4 k_{1}^{3} k_{2} A_{1}-3 k_{1}^{2} k_{2} B_{1}^{2}+2 k_{1}^{3} k_{2} A_{1}+3 k_{1}^{2} k_{2} A_{1}^{2}=0 .
$$

By solving the above system, we find Case 1:

$$
\begin{aligned}
& A_{0}=A_{0}, \quad A_{1}=-k_{1}, \quad B_{1}=-\mathrm{i} k_{1} \\
& \nu=\frac{k_{1}^{3} k_{2}-k_{3}^{2}}{k_{1}+k_{2}+k_{3}},
\end{aligned}
$$

Case 2:

$$
\begin{aligned}
& A_{0}=A_{0}, \quad A_{1}=-k_{1}, \quad B_{1}=\mathrm{i} k_{1} \\
& \nu=\frac{k_{1}^{3} k_{2}-k_{3}^{2}}{k_{1}+k_{2}+k_{3}},
\end{aligned}
$$

\section{Case 3:}

$$
\begin{aligned}
& A_{0}=A_{0}, \quad A_{1}=-2 k_{1}, \quad B_{1}=0 \\
& \nu=\frac{4 k_{1}^{3} k_{2}-k_{3}^{2}}{k_{1}+k_{2}+k_{3}} .
\end{aligned}
$$

According to Eqs. (69), (71)-(73) and inserting the result into the wave transformation (66), we obtain the following solutions for KP equation, which are in terms of hyperbolic functions:

From (69), we obtain

$$
\begin{aligned}
& u_{1}(x, y, z, t)= \\
& -\mathrm{i} k_{1} \operatorname{sech}\left(k_{1} x+k_{2} y+k_{3} z-\frac{k_{1}^{3} k_{2}-k_{3}^{2}}{k_{1}+k_{2}+k_{3}} t\right) \\
& -k_{1} \tanh \left(k_{1} x+k_{2} y+k_{3} z-\frac{k_{1}^{3} k_{2}-k_{3}^{2}}{k_{1}+k_{2}+k_{3}} t\right) \\
& +A_{0}, \\
& u_{2}(x, y, z, t)= \\
& \mathrm{i} k_{1} \operatorname{sech}\left(k_{1} x+k_{2} y+k_{3} z-\frac{k_{1}^{3} k_{2}-k_{3}^{2}}{k_{1}+k_{2}+k_{3}} t\right) \\
& -k_{1} \tanh \left(k_{1} x+k_{2} y+k_{3} z-\frac{k_{1}^{3} k_{2}-k_{3}^{2}}{k_{1}+k_{2}+k_{3}} t\right) \\
& +A_{0} \text {. }
\end{aligned}
$$

and

From (70), one can see

$$
\begin{aligned}
& u_{3}(x, y, z, t)= \\
& \quad-2 k_{1} \tanh \left(k_{1} x+k_{2} y+k_{3} z-\frac{4 k_{1}^{3} k_{2}-k_{3}^{2}}{k_{1}+k_{2}+k_{3}} t\right) \\
& +A_{0} .
\end{aligned}
$$

In the following, we have to do with the physical explanation and the graphical representation of the determined solutions of new $(3+1)$-dimensional generalized KP equation. The recovered solutions from case 1 , case 2 and case 3 , respectively, are hyperbolic function solutions. For $k_{1}=2, k_{2}=k_{3}=1, y=z=0.1$ and $A_{0}=5$, the solutions $u_{1}(x, y, z, t), u_{2}(x, y, z, t)$ and $u_{3}(x, y, z, t)$ are the travelling wave solutions with the interval $-10 \leq x, t \leq 10$ for case 1 , case 2 and case 3 , which are represented by Figs. 1, 2, and 3. The following representations of Figs. $1-3$ are obtained by the aid of Maple. 


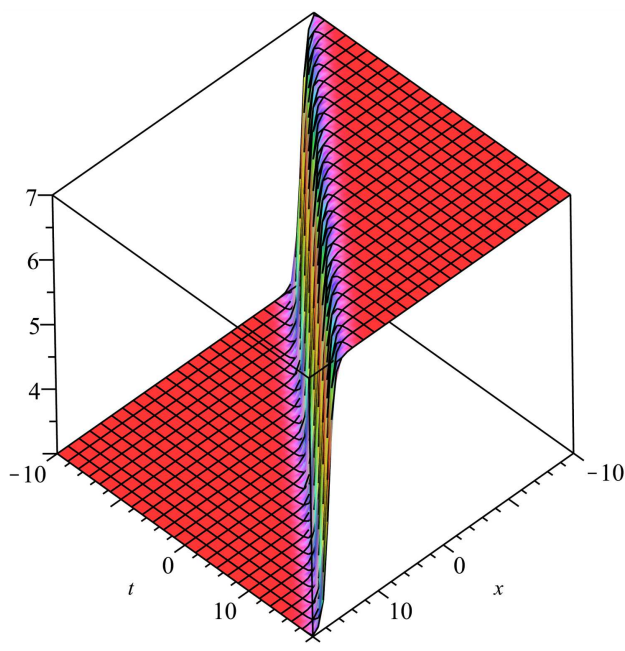

Fig. 1. The solution $u_{1}(x, y, z, t)$ for new $(3+1)$ dimensional generalized KP equation.

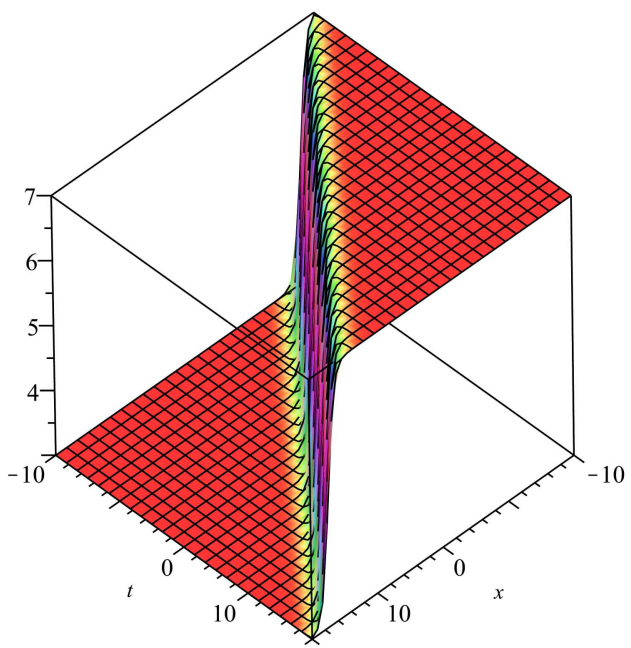

Fig. 2. As in Fig. 1, but for the solution $u_{2}(x, y, z, t)$.

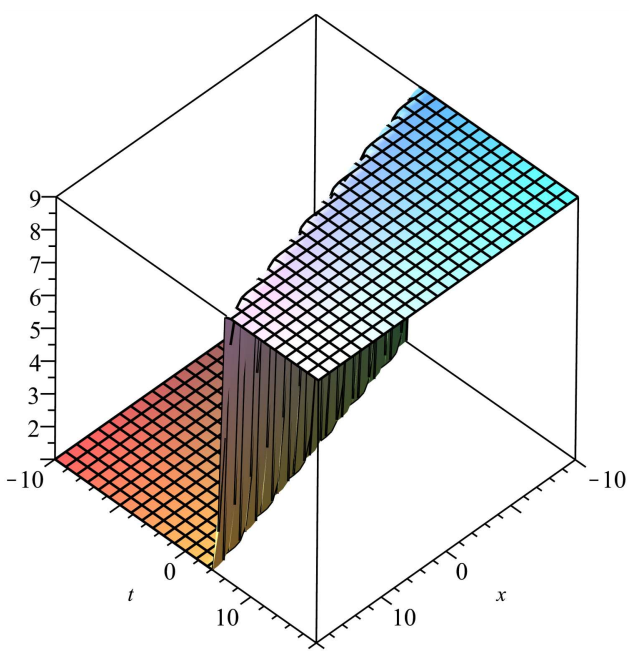

Fig. 3. As in Fig. 1, but for the solution $u_{3}(x, y, z, t)$.

\section{Conclusions}

This paper discussed a version of KP equation from $(3+1)$-dimensions that is studied in mathematical physics. Lie symmetry analysis, sub-ODE method, series solutions approach, sine-Gordon expansion method as well as traveling wave hypothesis are all applied to extract exact solutions to this KP equation. Solitary waves, shock waves as well as other forms of solutions are listed. These solutions are extremely useful in wide spread areas. The results of this paper will be of great asset in further future research. This model will be studied later with time-dependent coefficients, fractional temporal evolution, as well as stochastic coefficients. These just form a tip of the iceberg. Those results will be reported in future.

\section{Acknowledgments}

This work of Qin Zhou was supported by the National Natural Science Foundation of China (grant nos. 11705130 and 1157149), this author was also sponsored by the Chutian Scholar Program of Hubei Government in China. This work of Aimin Pan was supported by the Young Foundation of Wuhan Donghu University under the grant no. 2018dhzk003. The authors declare that they have no conflict of interest.

\section{References}

[1] A.M. Wazwaz, S.A. El-Tantawy, Nonlinear Dyn. 84, 1107 (2016).

[2] N.A. Kudryashov, Commun. Nonlinear Sci. Numer. Simulat. 14, 3507 (2009).

[3] A.H. Bhrawy, M.A. Abdelkawy, A. Biswas, Commun. Nonlinear Sci. Numer. Simulat. 18, 915 (2013).

[4] A. Biswas, A. Ranasinghe, Appl. Math. Comput. 214, 645 (2009).

[5] A. Biswas, A. Ranasinghe, Appl. Math. Comput. 217, 1771 (2010).

[6] H. Triki, B.J.M. Sturdevant, T. Hayat, O.M. Aldossary, A. Biswas, Can. J. Phys. 89, 979 (2011).

[7] G. Ebadi, N.Y. Fard, H. Triki, A. Biswas, Can. J. Phys. 17, 280 (2012).

[8] A.M. Wazwaz, Nonlinear Dyn. 83, 591 (2016).

[9] A.M. Wazwaz, S.A. El-Tantawy, Nonlinear Dyn. 88 , 3017 (2017).

[10] A.M. Wazwaz, Phys. Scripta, 89(9), 095206 (2014).

[11] A.M. Wazwaz, Int. J. Comput. Math. 82(2), 235 (2005).

[12] M. Wei, J. Cai, J. Appl. Math. Phys. 5, 715 (2017).

[13] M. Wei, J. Cai, Kinet. Relat. Mod. 6, 898 (2013).

[14] M.J. Ablowitz, D.E. Baldwin, Phys. Rev. E 86, 036305 (2012).

[15] C. Wang, Z. Dai, Appl. Math. Comput. 235, 332 (2014). 